\title{
Indica rice genome assembly, annotation and mining of blast disease resistance genes
}

\author{
H. B. Mahesh ${ }^{1,2,4}$, Meghana Deepak Shirke ${ }^{1,5}$, Siddarth Singh ${ }^{3}$, Anantharamanan Rajamani', Shailaja Hittalmani ${ }^{2}$, \\ Guo-Liang Wang ${ }^{4}$ and Malali Gowda ${ }^{1,6^{*}}$
}

\begin{abstract}
Background: Rice is a major staple food crop in the world. Over $80 \%$ of rice cultivation area is under indica rice. Currently, genomic resources are lacking for indica as compared to japonica rice. In this study, we generated deep-sequencing data (Illumina and Pacific Biosciences sequencing) for one of the indica rice cultivars, HR-12 from India.

Results: We assembled over $86 \%$ (389 Mb) of rice genome and annotated 56,284 protein-coding genes from HR-12 genome using Illumina and PacBio sequencing. Comprehensive comparative analyses between indica and japonica subspecies genomes revealed a large number of indica specific variants including SSRs, SNPs and InDels. To mine disease resistance genes, we sequenced few indica rice cultivars that are reported to be highly resistant (Tetep and Tadukan) and susceptible (HR-12 and Co-39) against blast fungal isolates in many countries including India. Whole genome sequencing of rice genotypes revealed high rate of mutations in defense related genes (NB-ARC, LRR and PK domains) in resistant cultivars as compared to susceptible. This study has identified R-genes Pi-ta and Pi54 from durable indica resistant cultivars; Tetep and Tadukan, which can be used in marker assisted selection in rice breeding program.
\end{abstract}

Conclusions: This is the first report of whole genome sequencing approach to characterize Indian rice germplasm. The genomic resources from our work will have a greater impact in understanding global rice diversity, genetics and molecular breeding.

Keywords: O. sativa, Genome sequencing, Indica, Illumina, Pacific Biosciences, SSRs, SNPs, Blast resistance

\section{Background}

Rice (Oryza sativa L.) is a staple food for more than half of the world's population. India is believed to be a center of origin and diversity of rice. India is the second most rice producer in the world and $65 \%$ of its population depends on rice as a staple food. Rice is a model cereal crop with small genome size, short generation time, diploid $(2 \mathrm{n}=24)$ and amenable for genetic manipulation. Due to its global importance, several genomes of rice cultigen including japonica (Nipponbare [1]), indica (93-11 [2] and IR64 [3]) and aus (Kasalath [4]) have been sequenced. Currently gold standard assembly and annotation are available for japonica

* Correspondence: malalig@ccamp.res.in; malalig@frlht.org

'Genomics Laboratory, Centre for Cellular and Molecular Platforms (C-CAMP),

National Centre for Biological Sciences (NCBS), Bengaluru 560065, India

${ }^{6}$ Genomics Discovery Program, School of Conservation, Life Science and

Health Sciences, TransDisciplinary University, Foundation of Revitalization of Local Health Traditions, Bengaluru 560064, India

Full list of author information is available at the end of the article rice. Although over $80 \%$ of rice cultivation in the world under indica rice cultivation however, genomic resources are lacking for this subspecies. Due to non-availability of proper genome assembly, indica genome studies are still using Nipponbare genome as a reference. This will introduce the potential bias in analysis and may not capture conclusive results at the nucleotide and chromosomal level for indica subspecies [3]. Thus, we and other researchers [3] believe that creating an indica reference rice genome is essential for genome-wide studies, which will enable genome assisted indica rice breeding program.

Here we report the de novo genome assembly and annotation of indica cultivar, HR-12. This cultivar was bred and released in India (http://www.drricar.org). It has good agronomic traits, but highly susceptible to blast disease [5], caused by Magnaporthe oryzae. Rice blast is a serious 
constraint in rice production and utilization of resistant (R) genes in variety development has become the most effective method of blast disease management. Sequencing of highly resistant and susceptible varieties will enable the identification of novel $\mathrm{R}$ genes and their deployment in rice breeding programme. In addition to HR-12, we resequenced whole genomes of three indica cultivars, which are highly susceptible (Co-39) and resistant (Tetep and Tadukan) to rice blast. Our indica rice sequencing efforts have complemented the global rice genomic resources, which eventually will help to characterize indica rice germplasm to identify genes for agronomically important traits including disease, pest and yield attributing traits.

\section{Methods}

\section{Indica rice cultivars and genome size estimation}

The HR-12 (Himmatsagar Rice-12) was derived from Raja Hansa (http://inger.irri.org). Subsequently HR-12 was used to develop Hamsa (HR-12 x TN-1) and Tellahamsa (HR-12 x TN-1) rice varieties (Additional file 1). The Co-39 variety was developed by crossing Culture340 and Kannagi. These two varieties have been widely used as susceptible checks in rice blast screening nursery. Tetep and Tadukan are used as resistance checks, and donor parents for blast resistance breeding, however the genealogy information is not available. The seeds of HR12, Co-39, Tetep and Tadukan were sown in a PVC pot containing red earth and fertilizers. Twenty one days old leaves from Co-39, HR-12, Tetep and Tadukan were collected and chopped into pieces in nuclear isolation buffer (Hypotonic Propidium Iodide, $50 \mu \mathrm{g} / \mathrm{mL}$ in $3 \mathrm{~g} / \mathrm{L}$ TriSodium citrate Dihydride containing $0.05 \%(\mathrm{v} / \mathrm{v})$ of Nonidet P-40 containing $2 \mathrm{mg} / \mathrm{mL}$ RNase A) and samples were processed as per the protocol suggested by Krishna [6]. Debris was filtered and stained nuclei were analysed using BD FACS at Central Imaging and Flow Cytometry Facility (CIFF), C-CAMP, NCBS, Bengaluru, India. Values of rice nuclear DNA was estimated by comparing rice nuclear peak on the linear scale with the peak for Pisum sativum included as an internal standard.

\section{Nucleic acid isolation}

Genomic DNA was isolated from four (HR-12, Co-39, Tetep and Tadukan) varieties as per the manufacturer's instruction (DNAeasy Plant Mini Kit, Cat \# 69104, USA). DNA quality was assessed by Nanodrop and DNA was quantified using Qubit (Applied Biosystems).

\section{Paired-end (PE) and matepair (MP) library preparation, and Illumina sequencing}

One micro gram of genomic DNA was fragmented in the range of 300 to 400 bases using ultra Sonicator (S220, Covaris, USA). Then PE library of HR-12, Co-39, Tetep and Tadukan samples were prepared using Tru
Seq DNA sample preparation kit v2 (Catalog No: FC121-2001, Illumina) as per the manufacture's instruction. PE libraries were sequenced using Illumina HiSeq1000 and the length of sequence was $101 \mathrm{nts}$ from both ends of the fragment. The MP library (insert size upto $12 \mathrm{~Kb}$ ) was prepared only for HR-12 sample by Nextera MP sample preparation kit (catalog No.: FC-132-1001, Illumina) and sequenced $2 \times 51$ nts by Illumina HiSeq1000.

\section{Strand-specific RNA seq library preparation and sequencing}

Total RNA was isolated from HR-12 leaves using Direct-zol RNA MiniPrep kit (Catalog No. R2050, Zymo Research) and RNA integrity and quantity was assessed by Bioanalyzer using Agilent RNA 6000 nano chip. TruSeq stranded total RNA library preparation kit v2 (Catalog No.: RS-1222201) from Illumina was used to prepare strand-specific RNA sequencing (ssRNA-seq) library by following manufacture's instruction. The ssRNA-seq library was sequenced 2x101 nts by Illumina HiSeq1000.

\section{PacBio library preparation and sequencing}

Around 20 micrograms of high quality genomic DNA was sheared using Hydroshear. The Bluepippin was used to select $20 \mathrm{~Kb}$ double-stranded DNA fragments. Then, DNA fragments were end repaired and ligated with universal hairpin adapters. Subsequent steps were followed as per the manufacture's instruction to prepare SMRTbell library. The library was sequenced in PacBio RS SMRT instrument.

\section{Illumina data output and data preprocessing}

The low quality bases (quality less than Q30 or the accuracy less than $99.99 \%$ of the base called) and adapter sequence contaminations in raw reads of Illumina sequencing (PE, MP and ssRNA-seq) was processed using FASTX-Toolkit (http://hannonlab.cshl.edu/fastx_toolkit/ index.html).

Short read (Illumina) de novo genome assembly of HR-12 The quality processed Illumina reads (PE and MP reads) were used for de novo assembly using three de novo genome assemblers viz., Velvet [7], SOAPDenovo2 [8] and MaSuRCA [9]. The quality of genome assemblies were assessed using QUAST [10].

\section{Gap filling of short read assembly using PacBio long reads, contigs scaffolding and anchoring}

Raw PacBio reads were used for gap filling and to upgrade short read assembly using PBJelly pipeline [11]. Gap filled assembly was further scaffolded by L_RNA_ scaffolder [12] using transcripts assembled by Trinity [13]. The pseudomolecules of HR-12 were constructed by anchoring HR-12 contigs on to pseudomolecules of Nipponbare genome (version 7.0) with ABACAS [14]. 
The synteny map of rice genomes was generated with default parameters by SyMAP [15]. The genome completeness of short read and gap filled assemblies were checked by CEGMA [16].

\section{Gene prediction and functional annotation of HR-12 genome}

The HR-12 pseudomolecules were subjected for gene prediction with MAKER-P [17] version 2.31 .6 by providing expressed sequences (trinity assembled HR-12 transcripts, ESTs, cDNA and mRNA) of Oryza (NCBI). Protein domain structures and gene ontology (GO) terms were assigned using InterProScan5 software [18]. Functional annotation of genes was done by searching homology against rice protein sequences of SwissProt (http://www. uniprot.org) using BLASTp alignments with an e-value threshold of $1^{\mathrm{e}-10}$. The synonymous (Ks) and nonsynonymous (Ka) substitution rate was calculated using NG [19] method in Ka/Ks calculator [20].

\section{Annotation of HR-12 specific genes}

Nipponbare (version 7.0) and 93-11 gene models were downloaded from MSU rice genome FTP site (ftp:// ftp.plantbiology.msu.edu/pub/data/Eukaryotic_Projects/ o_sativa/annotation_dbs/pseudomolecules/version_7.0/ all.dir/) and BGI (http://rice.genomics.org.cn/rice/ index2.jsp) site, respectively. The homology search was carried out with BLASTp using Nipponbare and 93-11 proteins as subject and HR-12 proteins as a query; the evalue cutoff was set to $1^{\mathrm{e}-10}$.

\section{Repeat identification and prediction of Simple Sequence Repeats (SSRs)}

De novo repeat prediction was performed using Repeat Masker 4.0.5 (http://www.repeatmasker.org) using Oryza sativa repeat library in Repbase as a reference. The SSRs were predicted using Microsatellite Identification tool [21]. Rice SSR markers in Gramene website (http:// archive.gramene.org/markers/microsat/all-ssr.tab) were used for performing an electronic PCR [22] (e-PCR) to check polymorphic and novel SSRs in HR-12 genome.

\section{Identification of SNPs, InDels and functional annotation of variants}

We used Illumina data of HR-12, Co-39, Tetep and Tadukan for variant analysis. The high quality (Q30) Illumina reads were mapped to Nipponbare genome using Burrows-Wheeler Aligner (BWA) V0.7.9a [23]. Alignments with mapping quality (' $\mathrm{q}$ ' option in samtools) $<60$ (Phred-scaled) were filtered using SAMtools [24]. Duplicate reads were removed using Picard tool v1.115 (http://broadinstitute.github.io/picard/). We performed local realignment around InDels to correct mapping related artifacts using InDelRealigner tool in GATK
V3.3-0. Then base quality recalibration was performed using BaseRecalibrator tool in GATK V3.3-0. Variant calling was performed with a minimum Phred-scaled confidence threshold of 30, and a minimum Phredscaled confidence threshold for emitting variants at 10 using HaplotypeCaller in GATK V3.3-0 across all four samples $[25,26]$. Functional annotation and genetic consequences (effect on gene) of common variants (SNPs and InDels) of susceptible and resistant varieties was annotated using SnpEff tool V4.1b [27]. The Nipponbare genome version 7.0 was used as a reference to annotate SNPs and InDels.

\section{Mining of blast disease resistance genes in indica cultivars}

The sequences of twenty-two cloned blast $R$-genes (NCBI) were subjected to BLASTp alignment (e-value cutoff $1^{\mathrm{e}-10}$, minimum $70 \%$ identity and query coverage) with proteins sequences of HR-12, Co-39, IR64 (http:// schatzlab.cshl.edu/data/rice/), Tetep, and Tadukan.

\section{Results and discussion}

De novo short read HR-12 genome assembly

The genome of indica rice cultivar, HR-12 was assembled using combination of short reads ( $\mathrm{PE}$ and MP libraries) from Illumina and long reads (Additional file 2) from Pacific Biosciences (Additional file 3). Initially, the Illumina short reads were assembled using three de novo assemblers Velvet, SOAPdenovo2 and MaSuRCA. Among three assemblers, MaSuRCA covered highest genome size of $340.12 \mathrm{Mb}$ and it had least number of N's as compared to other two assemblers. All three assemblers resulted comparatively lower contig N50 (Additional file 4), which might result due to inherent disadvantages of short read assemblies, such as poor repeat resolution [3, 28], missing exons and genes and genes split between scaffolds [29]. Less number of contigs in Velvet [7] and SOAPDenovo2 [8] assemblies were because of higher number of ' $N$ 's in the assembly, which were used to merge adjacent contigs into scaffolds (Additional file 4). Based on quality assessment by QUAST, MaSuRCA assembly was chosen for further analysis since it showed more improvement with respect to genome coverage (74.80\%), lower number of gaps (47479) and higher number of genes covered (48428) (Additional file 5).

Improvement of short read indica rice genome assembly using PacBio long reads

To improve assembly quality and overcome the limitations of short read assembly, HR-12 genome was sequenced using PacBio RS SMRT platform with 20x coverage. The PacBio reads were used to fill the gaps in the short read assembly (Fig. 1) which improved HR-12 genome with respect to genome size, N50 and number 


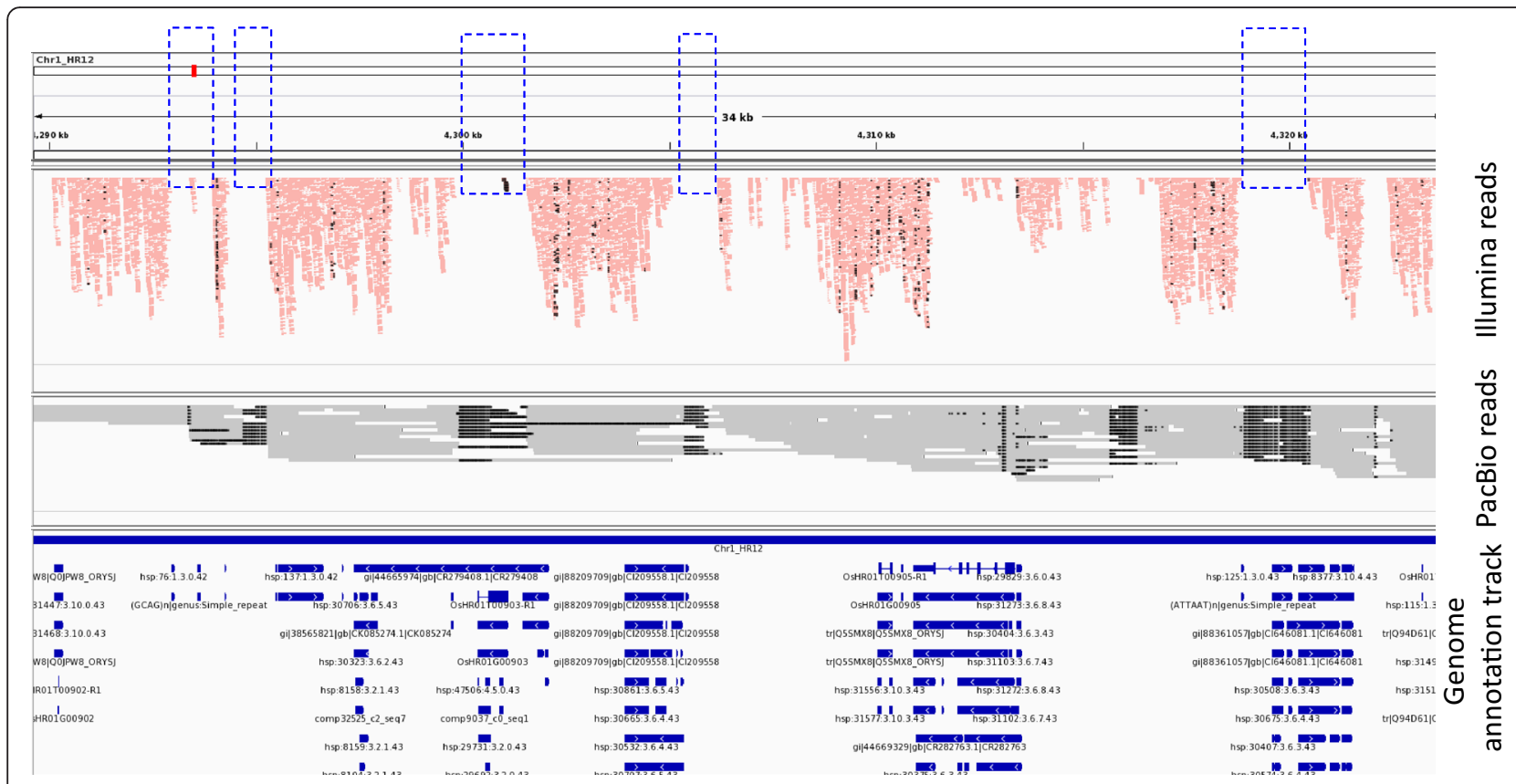

Fig. 1 Diagram depicting the assembly gaps closed by PacBio long reads. Gaps in short read assembly are shown in broken rectangles and gene models are represented in genome annotation track

of contigs/scaffolds. The genome assembly size was increased by $49.52 \mathrm{Mb}$ which accounts for over $86 \%$ of estimated HR-12 genome (454 Mb). The average genome size of four indica cultivars was found to be higher (457 Mb) as compared to previous report [30] (Additional files 6 and 7). The N50 of gap filled assembly was increased from 6.82 to $26.46 \mathrm{~Kb}$. Similarly, the number of scaffolds was reduced from 98939 to 61001 (Additional file 4). The Core Eukaryotic Genes (CEG) mapping approach has resulted $82.26 \%$ and $94.35 \%$ genes in short read and gap filled assemblies, respectively. According to our data, the gap filled indica rice, HR-12 genome assembly is much better with respect to genome size, number of genes, scaffold N50 and genome completeness as compared to other rice genomes including indica (93-11 and IR64), aus (Kasalath) and japonica (Nipponbare) (Table 1). The overall assembly quality comparison with other published genomes reiterated the importance of PacBio long reads in generating gold standard genomes for complex eukaryotic organisms. In a recent study, reference mapping of sequence reads of 50 rice accessions to japonica (Nipponbare), indica (93-11) and aus (Kasalath) genomes showed higher rate of mapping to Kasalath followed by $93-11$ and Nipponbare genomes [4]. This indicates that 93-11 genome is not well assembled and annotated [3, 4].

\section{HR-12 contigs ordering on Nipponbare chromosomes} Ordering and orienting of contigs/scaffolds onto pseudomolecules has facilitated identification of gaps, closure of gaps and also comparative whole genome analyses. We scaffolded HR-12 contigs by utilizing stranded RNA sequencing data, which reduced scaffold numbers from 61001 to 59692 . Over $73 \%$ of HR-12 contigs (43781 out of 59692 contigs) anchored onto pseudomolecules of Nipponbare with $80 \%$ sequence identity and remaining 15911 were unanchored. The unanchored contigs could be part of structural variants like insertions, inversions and translocations. The minimum and maximum contig length of unanchored contigs was 226 and $392500 \mathrm{bp}$, respectively. The N50 was 36469 bp with an average contigs length of $6642 \mathrm{bp}$. Nearly $55.40 \%$ (8815) and $60.34 \%$ (9600) of HR-12 contigs aligned to Nipponbare and 93-11 genomes with $100 \%$ query coverage and $80 \%$ sequence similarity, which confirmed that unaligned contigs were part of rice genome. Around $1.55 \%$ (247) of contigs did not show any alignment to Nipponbare genome indicating their absence in the reference genome.

The synteny map of HR-12, Nipponbare and 93-11 (Additional file 8) showed that most of the genomic blocks conserved across all three genomes with few translocations. Around $79 \%$ and $74 \%$ of HR-12 genome is in syntenic with Nipponbare and 93-11 genomes, respectively which restate that 93-11 genome is not well assembled.

\section{Gene prediction and functional annotation}

The gene prediction using MAKER-P in gap filled assembly of HR-12 genome yielded 56,284 protein-coding transcripts. We performed two-way comparison of genes 
Table 1 Comparison of whole genome sequencing of Oryza sativa subspecies

\begin{tabular}{|c|c|c|c|c|c|}
\hline Details & HR-12 (current study) & $93-11[2]$ & IR-64 [3] & Kasalath [4] & Nipponbare [1] \\
\hline Subspecies & indica & indica & indica & aus & japonica \\
\hline Sequence depth & $\begin{array}{l}\text { 73x (Illumina) and } \\
\text { 20x (PacBio) }\end{array}$ & $\begin{array}{l}4.2 x \text { (Sanger) and } \\
36 x \text { (Illumina) }\end{array}$ & 110x (Illumina) & $\begin{array}{l}\text { 6x (Roche 454) and } \\
\text { 148X (Illumina) }\end{array}$ & $\begin{array}{l}\text { 6x (Sanger), 62x (Illumina) } \\
\text { and 2.57x (Roche 454) }\end{array}$ \\
\hline Sequencing technologies & Illumina and PacBio & Sanger and Illumina & Illumina & Illumina and Roche 454 & Sanger, Illumina and Roche 454 \\
\hline Genome assembler(s) & MaSuRCA and PBJelly & RePS and SOAPDenovo & ALLPATHS-LG & $\begin{array}{l}\text { Celera Assembler and } \\
\text { String Graph Assembler }\end{array}$ & TIGR Assembler \\
\hline Genome size (Mb) & $389.77(389.60)$ & $374.55(359.41)$ & $345.9(321.2)$ & $401.14(328.25)$ & $374.47(374.31)$ \\
\hline No. of N's (Mb) & 0.17 & 15.14 & 24.7 & 72.89 & 0.17 \\
\hline $\begin{array}{l}\text { Total no. of } \\
\text { contigs/scaffolds }\end{array}$ & 59,692 & 50,231 & 26,160 & 51,550 & NA \\
\hline $\begin{array}{l}\text { a. Anchored } \\
\text { contigs/scaffolds }\end{array}$ & 43,781 & 35,415 & NA & 36,932 & NA \\
\hline $\begin{array}{l}\text { b. Unanchored } \\
\text { contigs/scaffolds }\end{array}$ & 15,911 & 14,816 & NA & 14,618 & NA \\
\hline $\mathrm{N} 50$ (Kb) & 28.5 & 6.69 & 22.2 & 13.73 & NA \\
\hline No. of genes & 56,284 & 40,464 & 37,758 & 53,662 & 55,986 \\
\hline CEG mapping (\%) & 94.35 & 95.97 & 94.35 & 95.97 & 95.97 \\
\hline
\end{tabular}

Value in parenthesis indicate genome size without ' $\mathrm{N}$ ' content $N A$ Not available, No. Number, $M b$ Mega bases, $K b$ Kilo bases N50 $50 \%$ of the contigs represent this contig length

CEG Core Eukaryotic Genes

from short read and gap filled assemblies, resulting into 31,933 genes with $100 \%$ identity. In addition, 2615 genes were annotated which were unique to gap filled assembly but absent in short read assembly. About $38.6 \%(21,736)$ of genes were fragmented in short read assembly as compared to gap filled assembly. This indicates that use of PacBio reads has significantly improved the indica rice genome annotation.

Genes of gap filled assembly were also compared with genes of Nipponbare and 93-11. There were 54,849 genes (e-value of $1^{\mathrm{e}-10}$ ) commonly present in both HR12 and Nipponbare. Similarly 54,130 genes were commonly present in HR-12 and 93-11. This indicates that over $\sim 97 \%$ of annotated rice genes from indica (93-11) and japonica (Nipponbare) were annotated in HR-12 gap filled assembly. Overall, 1950 genes were unique to HR-12 genome in comparison with Nipponbare and 9311 genomes (Additional file 9). These unique genes in HR-12 were annotated and classified as proteins of unknown function (96), uncharacterized proteins (1349) and proteins with known function (475). There were 30 resistance genes with NBS-LRR, LRR and kinase domains (Additional file 9).

\section{$\mathrm{Ka} / \mathrm{Ks}$ analysis for annotated proteins}

To determine the evolutionary selection pressure on proteome between indica (HR-12 and 93-11) and japonica (Nipponbare) genomes, synonymous amino acids substitution rates (Ks) and non-synonymous amino acids substitution rates $(\mathrm{Ka})$ were calculated. The $\mathrm{Ka} / \mathrm{Ks}$ ratio can reflect the selection pressure between gene pairs (homologs) caused by evolutionary forces like natural mutations. This homolog group analyses resulted 3424 and 5527 gene pairs between indica-indica (HR-12 v/s 93-11), and indica-japonica (HR-12 v/s Nipponbare), respectively. Homolog groups were classified into three categories based on $\mathrm{Ka} / \mathrm{Ks}$ ratio with probability value of $<0.05$ (Fisher exact test). The indica-indica homologs comparison yielded 32 and 3392 genes being positively and negatively selected with probability value of $<0.05$ (Fisher exact test), respectively. Similarly, indica-japonica homologs comparison showed 29 and 5498 genes were under positive $(\mathrm{Ka} / \mathrm{Ks}$ ratio $>1)$ and negative $(\mathrm{Ka} / \mathrm{Ks}$ ratio $<1)$ selection, respectively (Additional file 10).

\section{Genome-wide comparison of SSRs and SNPs in sequenced genomes}

The SSRs are repetitive DNA sequences used as codominant molecular markers to determine genetic diversity and mapping of genes/QTLs. Identification of SSRs in genome sequences will increase the availability of more genomic resources. In total, 114508, 145371, 135501 and 141177 SSRs were identified in Illumina HR-12 assembly, long read HR-12 assembly, Nipponbare and 93-11 genomes, respectively (Fig. $2 \mathrm{~b}$ and Additional file 11). Mono and di-nucleotide repeats were more in HR-12 genome as compared to Nipponbare and 93-11. The 'AT' (di-repeats) and AAG, AGG (tri-repeats) repeats were more predominant in HR-12 genome (Additional file 11). The 'AT' rich di-nucleotide repeats are reported to be most abundant in 

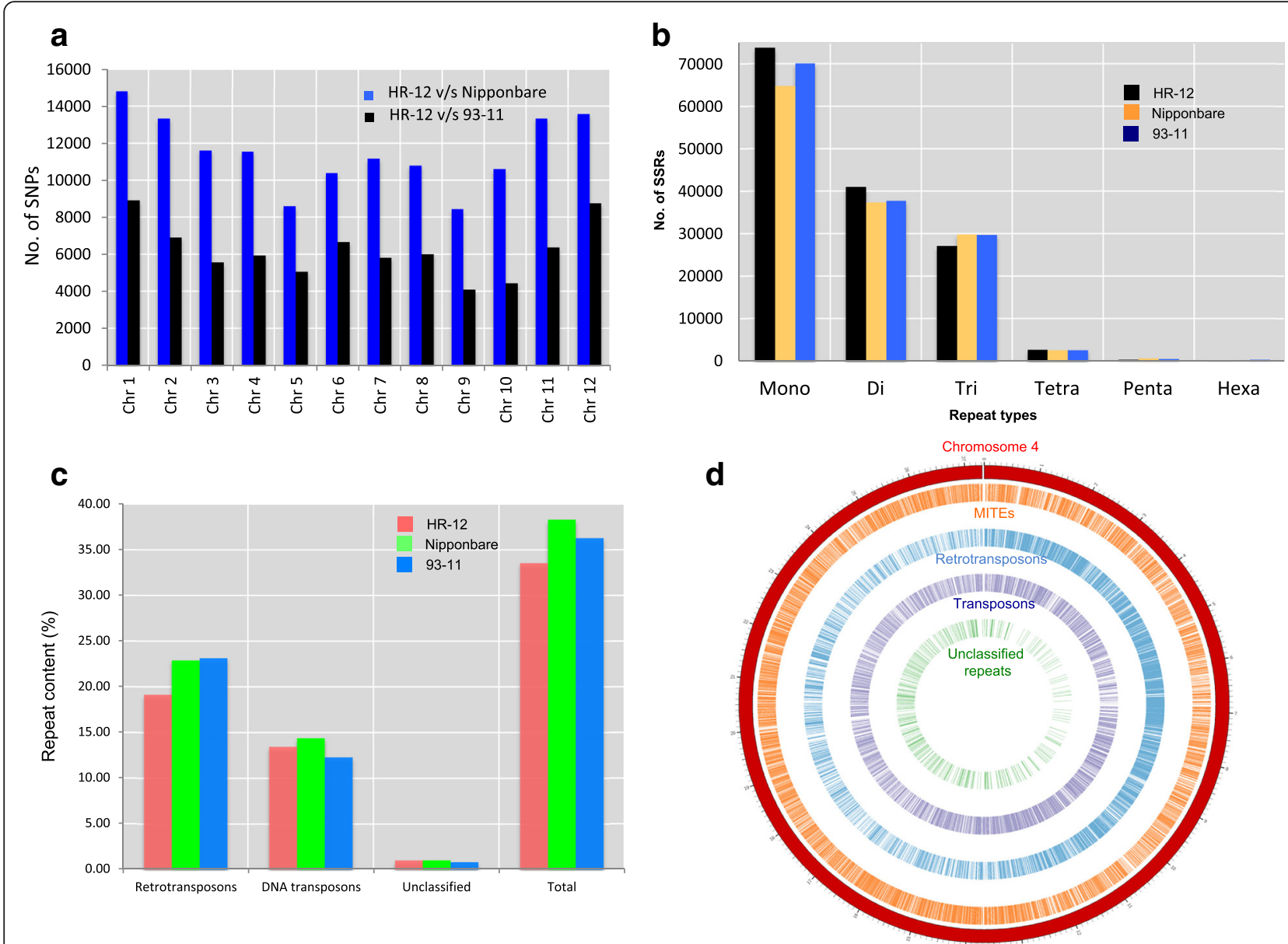

Fig. 2 Genome-wide comparison of HR-12, 93-11 and Nipponbare genomes for SNPs, SSRs and repeats. a Number of SNPs, (b) number of SSRs, (c) distribution of repeat elements and (d) density of repeats on chromosome 4 of HR-12 genome

rice genome as compared to other SSRs [31]. Among tetra- repeats; AAAC, AAAG, AAAT, AACC, AACG, AAGG, ACAT, ACGC, ACGG, ACGT and AGAT types were more abundant in HR-12 genome (Additional file 11) as compared to Nipponbare and 93-11 genomes.

More than 19000 SSRs are available in the Gramene database (http://archive.gramene.org/markers/microsat/ all-ssr.tab). These sequences were used for e-PCR analysis in HR-12, Nipponbare and 93-11 genomes. There were 11296 (58.55), 16144 (83.68) and 13429 (69.61) SSRs mapped to HR-12, Nipponbare and 93-11 genomes, respectively. This indicates that publicly available SSR markers were skewed towards japonica genome. Among 11296 SSRs from HR-12 assembly, 1095 SSRs were monomorphic and 10201 SSRs were polymorphic across three genomes. The pair-wise comparison of polymorphic SSRs has yielded 8914 polymorphic SSRs between HR-12 and Nipponbare, and 8445 SSRs polymorphic between HR-12 and 93-11. As expected, SSRs were less polymorphic within indica rice (HR-12 and 93-11) (indica type) and highly polymorphic between japonica rice (Nipponbare). Among 11296 SSRs in HR-12, 1617 SSRs were unique to HR-12 genome (Additional file 12). These unique SSRs (1617) were compared with sequenced genomes of other indica varieties, Tetep and Tadukan. Out of these, 1260 SSRs were found in both Tetep and Tadukan, which resulted 209 SSR markers unique to HR-12 genome. Out of remaining 148 SSRs, 128 SSRs were common between HR-12 and Tetep but absent in Tadukan genome and 20 were common between HR-12 and Tadukan.

The comparison of Gramene SSRs in HR-12 (11296) and predicted SSRs (56560) revealed that all 11296 public SSRs were present in predicted SSRs. Elimination of publicly available SSRs in predicted SSRs of HR-12 yielded 47378 SSRs to be novel from in silico prediction for indica genome. Among these, 20547 di, 18760 tri, 1040 tetra, 332 penta and 6699 were complex SSRs. The conserved flanking regions (100 bp upstream and downstream from SSR motif) of 43834 and 44123 SSRs of HR-12 (47378) were mapped to Nipponbare and 93-11 
genomes, respectively. Whereas 3533, 3255 HR-12 SSRs were unique to HR-12 and did not map to Nipponbare and 93-11 genomes.

Genome-wide comparison of SNPs between HR-12 and 93-11, HR-12 and Nipponbare discerned higher level of polymorphism between indica-japonica comparison (Fig. 2a). These species-specific variations can be used for marker assisted breeding, positional cloning and evolutionary studies.

\section{Repeats in HR-12 genome}

Repetitive DNA sequence can account for substantial portion of the many eukaryotic genomes and lead into genome expansion, gene disruption and gene duplication. Total 34.9 \% repeats were found in HR-12 genome (gap filled assembly) as compared to Nipponbare (39.64\%) and 93-11 (37.62 \%) (Fig. 2c). Total interspersed repeats were the major elements (33.57\%), consisting of retro-elements (19.14\%), DNA transposons (13.44\%) and unclassified repeats $(1 \%)$. In case of retro-elements, long terminal repeat
(LTR) had a highest fraction (17.72 \%), followed by LINEs $(0.98 \%)$ and SINEs $(0.43 \%)$. In case of DNA transposons, tourist/harbinger elements content was more (2.84 \%), followed by Tc1-IS630-Pogo (2.61 \%), hobo-Activator (0.52 \%), En-Spm (0.43 \%) and MuDR-IS905 (0.14 \%). Similar trend of repeat distribution was observed in case of Nipponbare and 93-11 genomes (Additional file 13). Chromosome-wise repeat distribution (Additional file 14) displayed highest repeat content of $39.60 \%$ on chromosome 4 and the lowest of $31.74 \%$ on chromosome 3 . The overall distribution of retro-transposons, DNA transposons and Miniature Inverted-repeat Transposable Elements (MITEs) in HR-12 genome for chromosome 4 is represented in Fig. 2d.

\section{Whole genome sequencing of blast susceptible and resistant indica rice varieties}

We sequenced indica cultivars, which are highly resistant (Tetep and Tadukan) and susceptible (HR-12 and Co-39) to rice blast disease caused by Ascomycetes pathogen

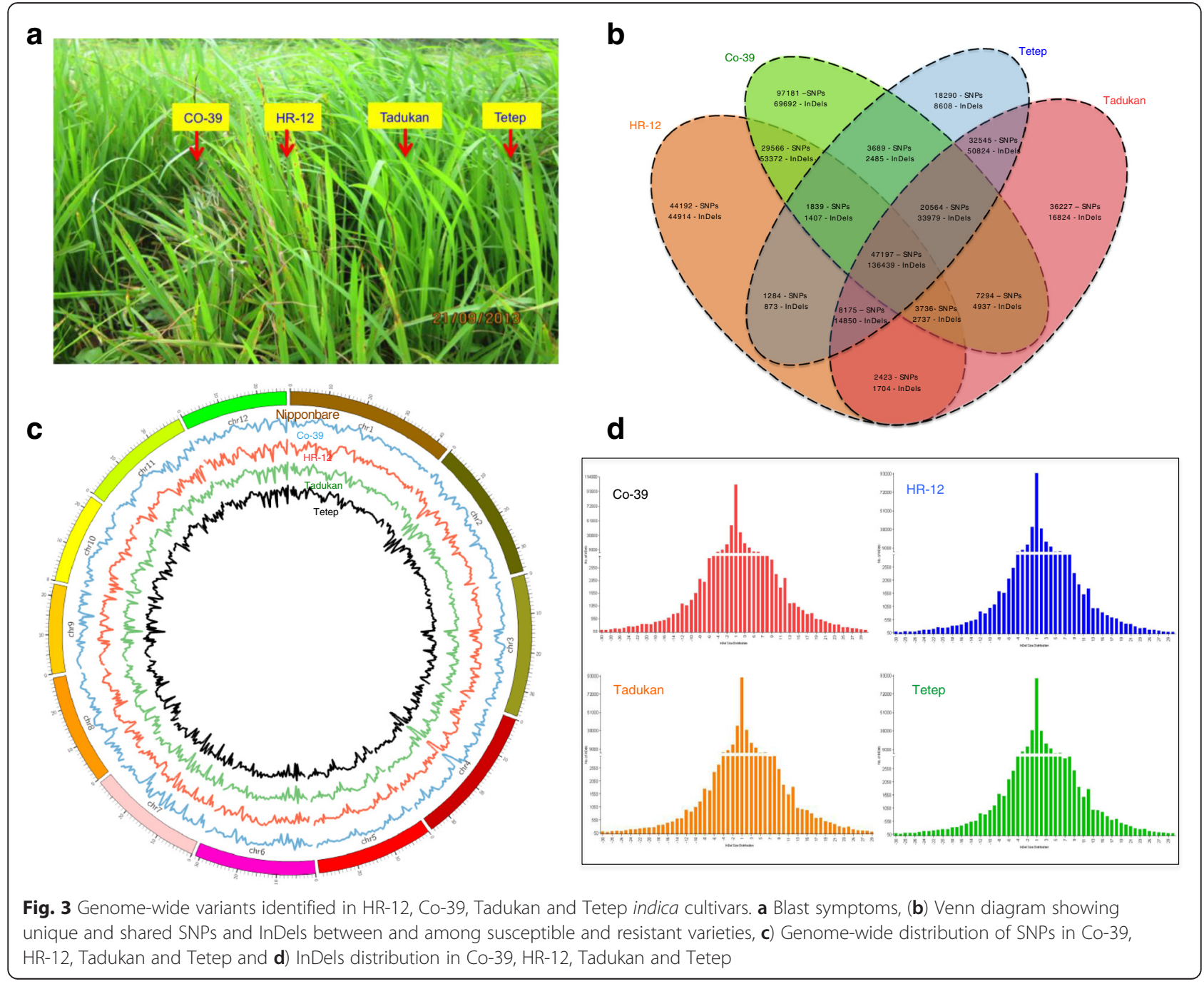


Magnaporthe oryzae (Fig. 3a). Mapping of sequence reads to Nipponbare genome enabled us to identify large number of variants in Co-39 (211066 SNPs and 305048 InDels), HR-12 (138412 SNPs and 256296 InDels), Tadukan (158161 SNPs and 262294 InDels) and Tetep (133583 SNPs and 383048 InDels) (Fig. 3c). Large fraction of SNPs and InDels were found in upstream and downstream regions of annotated genes (Additional file 15). The transition to transversion (Ts/Tv) ratio was $1.69,1.58,1.61$ and 1.58 in Co-39, HR-12, Tetep and Tadukan, respectively. The SNPs were located at the intervals of 1764, 2692, 2791 and 2357 nts in the genomes of Co-39, HR-12, Tetep and Tadukan, respectively. Similarly, the InDel rate was per 1207, 1449, 1493 and 1419 bases in Co-39, HR-12, Tetep and Tadukan, respectively. One base insertions $(+1)$ and deletions $(-1)$ were more [32] as compared to other types of InDels (Fig. 3d).

Among blast disease susceptible varieties (HR-12 and Co-39), 29566 SNPs and 53372 InDels and among resistant varieties (Tetep and Tadukan), 32545 SNPs and 50824 InDels were common (Fig. 3b). In blast susceptible varieties, 44192 SNPs and 44914 InDels were unique to HR-12 and 97181 SNPs and 69692 InDels were unique to Co-39. Similarly in blast resistant varieties, 36227 SNPs and 16824 InDels were unique to Tadukan where as 18290 SNPs and 8608 InDels were unique to Tetep.

\section{Functional annotation of variants in blast resistant and susceptible varieties}

Commonly occurring variants (29566 SNPs and 53372 InDels in susceptible; 32545 SNPs and 50824 InDels in resistant varieties) were annotated to know their functionality in the genome (Additional file 16). In total, 24431 and 27562 genes were mutated (SNPs and InDels) in susceptible and resistant varieties, respectively. We further classified these genes based on their protein domains and focused on genes involved in host defense mechanism (resistance genes) such as protein kinase (PK), nucleotide-binding adapter shared by APAF-1, R-proteins and CED-4 (NB-ARC), and leucine-rich repeats (LRR) domains. We observed defense related genes have accumulated more mutations (non- synonymous) in resistant varieties as compared to susceptible varieties (Table 2). There were 40 NB-ARC, 20 LRR, 93 PK genes were mutated (SNPs and InDels) in resistant varieties. In susceptible varieties, $16 \mathrm{NB}-\mathrm{ARC}, 17 \mathrm{LRR}$ and 67 PK genes were mutated. This functional annotation of variants is highly useful to develop PCR-based functional markers [33] to screen large set of rice germplasm and identify novel alleles of $R$ genes, enabling breeders to rapid introgression of resistance genes and gene pyramiding [34] to elite cultivars for durable blast resistance.

\section{Mining of blast disease resistant genes in indica rice varieties}

Till-date, 22 blast resistant $R$-genes cloned [35-39] from several rice varieties, which confers resistance against Magnaporthe isolates. To assess the spectrum of $\mathrm{R}$ genes in resistant (Tetep, Tadukan and IR64) and susceptible (Co-39 and HR-12) cultivars, we performed protein-protein alignment of R-genes. The R-genes such as Pi37, Pid2, Pid3, Pi25, Pish and Pi64 were conserved at structure level in all indica varieties, however, several SNPs and InDels were interrupted these genes. The Pi54 (Pikh) gene was present in Tadukan and Tetep but absent in Co-39, HR-12 and IR64. Pi-ta, a broad spectrum resistant gene was found in all four indica varieties. However, single amino acid substitution (Ala to Ser) was observed at 918 in Pi-ta protein, which reported to be responsible for determining resistance specificity [40]. We observed similar Ser substitution in Pi-ta protein in blast susceptible varieties (Co-39 and HR-12). In addition, we identified novel amino acid (Phe to Ser) substitution at 641 residues in all indica (Co-39, HR-12, IR64, Tadukan, Tetep) varieties (Fig. 4). Other genes such as Pib, Piz-t, Pik-m, Pi5, pi21, Pb1, Pik, Pik-p and Pi1 were absent in all indica varieties (Table 3$)$. Majority of R-genes were either mutated or fragmented in case of susceptible varieties. The $R$ genes in host and AVR genes in pathogen follow gene -for -gene hypothesis [41]. Survey of avirulent genes (AVR) in Magnaporthe population isolated from HR-12 from Southern India showed predominance of AVR-Pizt, AVR-Pita, AVR-Pik, AVR-Pii,

Table 2 Mutation in defense related genes in indica rice varieties

\begin{tabular}{|c|c|c|c|c|c|}
\hline \multirow{3}{*}{$\begin{array}{l}\text { Domain } \\
\text { Name }\end{array}$} & \multirow[t]{3}{*}{ Domain ID } & \multicolumn{4}{|c|}{ No. of genes mutated } \\
\hline & & \multicolumn{2}{|c|}{$\begin{array}{l}\text { Blast resistant varieties } \\
\text { (Tetep and Tadukan) }\end{array}$} & \multicolumn{2}{|c|}{$\begin{array}{l}\text { Blast susceptible varieties } \\
\text { (HR-12 and Co-39) }\end{array}$} \\
\hline & & SNPS & InDels & SNPS & InDels \\
\hline NB-ARC & PF00931 & 15 & 25 & 6 & 10 \\
\hline LRR & PF00560, PF08263, PF12799 & 3 & 17 & 2 & 15 \\
\hline Protein Kinase & $\begin{array}{l}\text { PF00069, PF07714, PF13947, PF03727, } \\
\text { PF00406, PF00781, PF00485 }\end{array}$ & 31 & 62 & 17 & 50 \\
\hline TOTAL & & 49 & 104 & 25 & 75 \\
\hline
\end{tabular}

NB-ARC Nucleotide-binding adaptor shared by APAF-1, R proteins, and CED-4 LRR Leucine Rich Repeats 


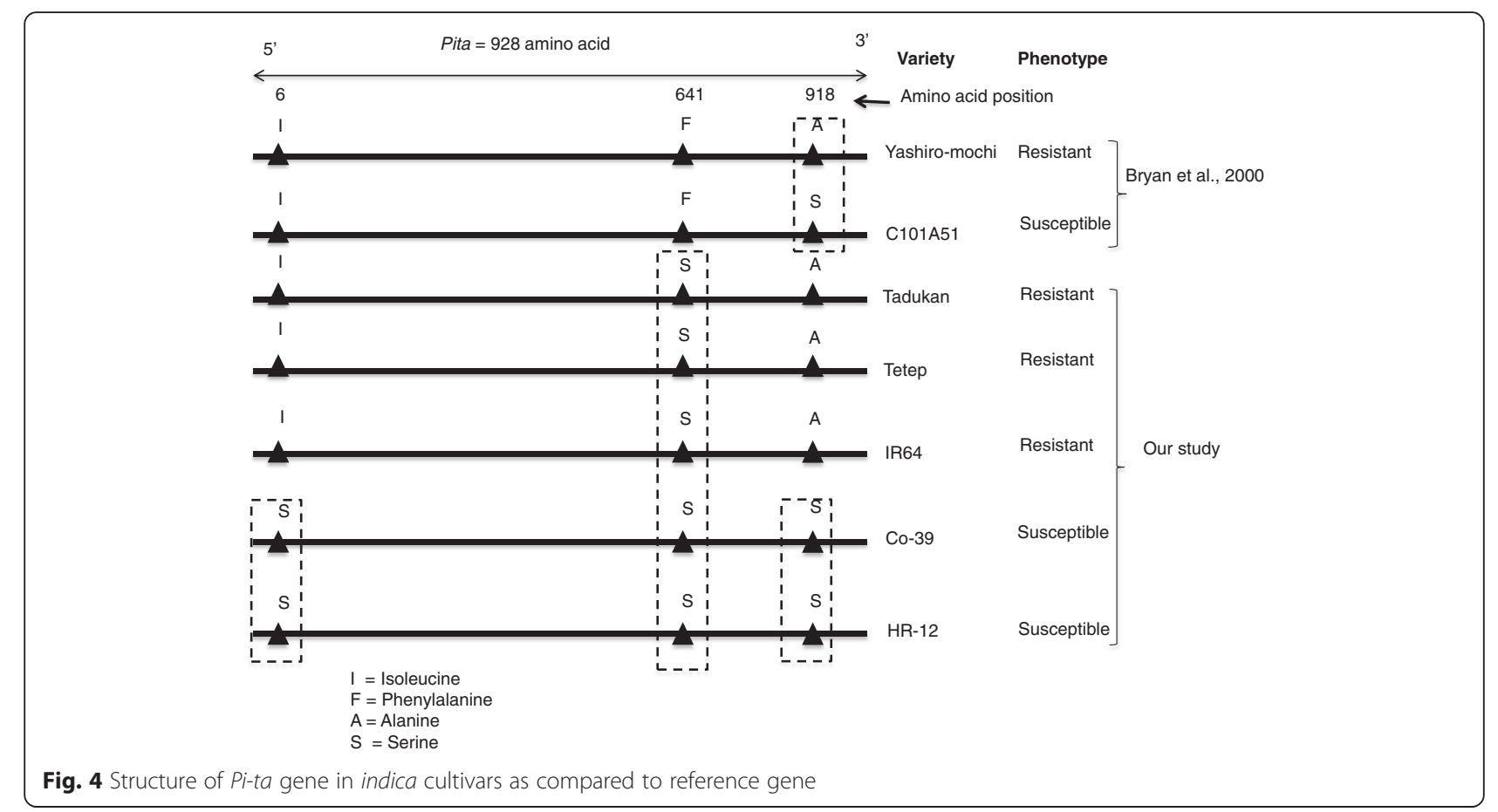

and AVR-Pia (Unpublished). The cognate R-genes like Piz-t, Pi-ta, Pik and Pia were absent in HR-12. Similarly, Magnaporthe isolates from Co-39 showed presence of AVR-Pizt, AVR-Pii and AVR-Pik and the cognate Rgenes, Piz-t and Pik genes were absent in Co-39 genome. Thus, study of $\mathrm{R}$ and AVR genes in the crop ecosystems play an important role to understand the evolution of new pathotypes and to design better plant breeding strategies.

\section{Conclusions}

High quality genomic resources are prerequisite for rice breeding. The available indica reference genomes 93-11 and IR64 were sequenced and assembled using short read sequencing technology. Inherent drawbacks of short read genome assemblies may cause alignment problem during reference mapping and study of structural variations. Due to non-availability of high quality indica reference genome, Illumina reads from 3000 rice accessions (indica, aus, tropical japonica, temperate japonica and aromatic) were mapped to Nipponbare genome to identify variants [42]. Although another indica genome IR64 [3] is sequenced, its chromosomes consist of complex recombination of fragments (mosaic) from the genealogy of more than 38 parents [43] including indica, japonica and wild species. With advent of third generation sequencing technology, it is possible to sequence plant genomes with higher accuracy and coverage. Thus, we report improved de novo assembly of indica cultivar HR-12 using combinatorial approach of short and long reads which covers over $86 \%$ of estimated genome size. The gap filling strategy with the help of long reads has improved the short read assembly with respect to genome size, repeat content, and number of protein coding genes. Whole genome comparison of HR-12, 93-11 and Nipponbare genomes revealed 1950 genes and 1617 SSRs unique to HR-12 genome with similar level of repeat content.

Rice blast is a major biotic stress in rice, which reduces yield significantly. To understand disease resistance genes, we sequenced highly resistant (Tetep and Tadukan) and susceptible (Co-39, HR-12) indica cultivars. Functional annotation of SNPs and InDels in indica cultivars showed higher non-synonymous substitutions in defense related genes [44, 45] containing NBARC, LRR and PK domains among resistant varieties indicating strong diversifying selection to confer resistance to fast evolving blast pathogen. Allele mining for resistance genes in all sequenced genomes showed presence/absence polymorphism and large number of structural variations. Most of the $\mathrm{R}$ genes were conserved in resistant cultivars with point mutations and InDels whereas loss of R-gene structure was noticed in susceptible cultivars. The broad-spectrum resistance in Tadukan, Tetep and IR64 could be attributed to presence of intact Pi-ta and Pi54 (absent in IR64). The rice cultivars, Tadukan and Tetep were found to be resistant against most of blast races across the globe [46, 47] and these have been used in IRRI for developing IR64. Previously, high rate of mutation in $\mathrm{R}$ genes have been 
Table 3 Distribution of cloned blast resistance genes in sequenced rice varieties

\begin{tabular}{|c|c|c|c|c|c|c|c|}
\hline 'R' gene & Donor Variety & Group & $\mathrm{HR}-12$ & Co-39 & Tetep & Tadukan & IR-64 \\
\hline Pib & Tohoku IL-9 & Japonica & - & - & - & - & M \\
\hline Pi-ta & Yashiro-mochi & Japonica & - & - & + & + & + \\
\hline Pi54 (Pik-h) & Tetep & Indica & - & - & + & + & - \\
\hline Pid2 & Digu & Indica & M & M & M & M & M \\
\hline Pig & $75-1-127$ & Indica & M & - & - & - & M \\
\hline Piz-t & Toride 1 & Japonica & - & - & - & - & - \\
\hline Pi37 & St.No. 1 & Japonica & M & M & M & M & M \\
\hline Pi36 & Kasalath & Indica & M & - & - & M & M \\
\hline Pik-m-TS1 & Tsuyuake & Japonica & - & - & - & - & - \\
\hline Pik-m-TS2 & Tsuyuake & Japonica & - & - & - & - & M \\
\hline pi21 & Owarihatamochi & Japonica & - & - & - & - & - \\
\hline Pit & Nipponbare & Japonica & M & - & M & - & M \\
\hline Pi5-1 & RIL260 & Japonica & - & - & - & - & M \\
\hline Pi5-2 & RIL260 & Japonica & - & + & - & - & - \\
\hline Pid3 & Digu & Indica & M & M & M & M & M \\
\hline$P b 1$ & St.No. 1 & Japonica & - & - & - & - & - \\
\hline Pish & Shin 2, Norin 22 & Japonica & M & M & M & M & M \\
\hline Pi25 & Gumei 2 & Japonica & M & M & M & M & M \\
\hline Pia (RGA4) & Sasanishiki, Aichi-asahi & Japonica & - & M & M & M & M \\
\hline Pik-p-1 & K60 & Japonica & - & - & - & - & M \\
\hline Pik-p-2 & K60 & Japonica & - & - & - & - & M \\
\hline Pik-1 & Kusabue & Japonica & - & - & - & - & M \\
\hline Pik-2 & Kusabue & Japonica & - & - & - & - & M \\
\hline Pi54rh & O. rhizomatis & Wild species & - & - & M & M & - \\
\hline Pi1-5 & LAC23, C101LAC & Indica & - & - & - & - & M \\
\hline Pi1-6 & LAC23, C101LAC & Indica & - & - & - & - & M \\
\hline Pi64 & Yangmaogu (YMG) & Japonica & M & M & M & M & M \\
\hline
\end{tabular}

$+=$ Present

- = Absent

$M$ Mutated but protein structure retained

reported in Rice (Xa21) [48] and Maize (Rp1) [49]. These allelic variations created by mutations will result in evolution of novel $\mathrm{R}$ genes/alleles and selection of genes that can recognize pathogen avirulence gene products. Identification of $\mathrm{R}$ genes/alleles is a prerequisite for effective utilization of genetic and genomic resources in modern plant breeding, which is driven by new genomics tools.

\section{Data availability}

The raw sequence reads deposited under NCBI Sequence Read Archive (SRA) accession numbers SRP067809 (HR-12), SRP067775 (Co-39), SRP067810 (Tetep), and SRP067808 (Tadukan). The whole genome shotgun projects have been deposited at DDBJ/EMBL/Genbank under the accessions AZTA02000000 (HR-12), LQHE 01000000 (Co-39), LQHG01000000 (Tetep), and LQHF 01000000 (Tadukan).

\section{Additional files}

Additional file 1: Genealogy of HR-12 variety. (PPTX $76 \mathrm{~kb}$ )

Additional file 2: Sequence data size for rice cultivars. (XLSX $15 \mathrm{~kb}$ )

Additional file 3: Analysis workflow followed for assembling Illumina and PacBio sequence reads of HR-12. (PPTX $129 \mathrm{~kb}$ )

Additional file 4: Short and long read assembly statistics of HR-12. (XLSX $16 \mathrm{~kb}$ )

Additional file 5: Assembly quality assessment by QUAST. (XLSX 16 kb) Additional file 6: Genome sizes of indica cultivars. (XLSX $16 \mathrm{~kb}$ ) 
Additional file 7: Frequency histograms of numbers of nuclei per channel as a function of relative fluorescence in Pisum (internal standard), Co-39, HR-12, Tetep and Tadukan. The ' $x$ ' and ' $y$ ' axes represents number of nuclei and linear fluorescence, respectively. (PPTX 298 kb)

Additional file 8: Comparison of syntenic blocks among HR-12, 93-11, and Nipponbare genomes. (a) syntenic blocks between HR-12 and 93-11. (b) syntenic blocks between HR-12 and Nipponbare and (c) syntenic blocks between HR-12, 93-11 and Nipponbare. (PPTX 3804 kb)

Additional file 9: Unique genes in HR-12 genome. (XLSX 97 kb)

Additional file 10: Gene under positive selection in indica-indica and indica-japonica comparisons. (XLSX $21 \mathrm{~kb}$ )

Additional file 11: Number of SSRs in Illumina short and PacBio long read assemblies (a), Number of tri and tetra SSRs of HR-12, 93-11 and Nipponbare genomes (b). Distribution of tetra type SSRs in HR-12 Nipponbare and 93-11 genomes (c). (PPTX 183 kb)

Additional file 12: Unique SSRs in HR-12 genome in comparison with Nipponbare and 93-11. (XLSX 133 kb)

Additional file 13: Repeat content in HR-12, Nipponbare and 93-11 genomes. (XLSX $19 \mathrm{~kb}$ )

Additional file 14: Chromosome-wise distribution of repeat elements in HR-12 genome. (XLSX 29 kb)

Additional file 15: Functional annotation of variants in CO-39, HR-12, Tetep and Tadukan varieties. (XLSX $18 \mathrm{~kb}$ )

Additional file 16: Number of effects of SNPs and InDels on various regions of genes. (XLSX $16 \mathrm{~kb}$ )

\section{Abbreviations}

Ala: Alanine; AVR: Avirulence; bp: basepair; BWA: Burrows-Wheeler Aligner; cDNA: Complementary DNA; ESTs: Expressed sequence tags; GO: Gene ontology; InDels: Insertions and Deletions; Kb: Kilobase; LINEs: Long Interspersed Nuclear Elements; LRR: Leucine-rich repeat; LTR: Long terminal repeat; Mb: Million bases; MITEs: Miniature Inverted-repeat Transposable Elements; MP: Mate pair; NB-ARC: Nucleotide-binding adaptor shared by APAF-1 R proteins, and CED-4; nts: Nucleotides; PE: paired-end; Phe: Phenylalanine; PK: Protein kinase; PVC: Polyvinyl chloride; QTLs: Quantitative trait loci; R: Resistance; Ser: Serine; SINEs: Short Interspersed Nuclear Elements; SNPs: Single nucleotide polymorphisms; SSRs: Simple sequence repeats; Ts: Transition; Tv: Transversion.

\section{Competing interests}

The author(s) declare that they have no competing interests.

\section{Authors' contributions}

HBM performed isolation of DNA and RNA, genome estimation, genome sequencing, assembly, annotation, variant calling and R-gene analyses of all indica cultivars, prepared all tables, figures and wrote the manuscript. MDS prepared the DNA for PacBio sequencing, KaKs and Repeat analysis and helped in writing manuscript. SS coordinated PacBio sequencing and performed gap filling of Illumina short read assembly and helped in writing manuscript. AR prepared CIRCOS figures. SH helped in writing manuscript. G-LW helped in writing manuscript. MG conceived the project, designed the experiment and wrote the manuscript. All authors read and approved the final manuscript

\section{Acknowledgements}

This work was supported by Department of Biotechnology (DBT), Government of India. We would like to acknowledge Texas A\&M, USA for awarding Monsanto's Beachell-Borlaug International Fellowship to H. B, Mahesh for his Ph.D programme. We are grateful to Genomics facility members; Chandana S, Shilpa S, Sahana S, Ashwini M, Sheetal A and Goutham for their help in sample preparation, sequencing and bioinformatics analyses. We thank Ram Laxman, Pacific Biosciences for helping in PacBio sequencing. We acknowledge Indian Institute of Rice Research (IIRR, formerly known as DRR) for providing Co-39, HR-12, Tetep and Tadukan seed material.

\section{Author details}

Genomics Laboratory, Centre for Cellular and Molecular Platforms (C-CAMP), National Centre for Biological Sciences (NCBS), Bengaluru 560065, India. ${ }^{2}$ Marker Assisted Selection Laboratory, Department of Genetics and Plant Breeding, University of Agricultural Sciences, Bengaluru 560065, India. ${ }^{3}$ Pacific Biosciences, Boon Lay Way, Singapore 609964, Singapore. ${ }^{4}$ Department of Plant Pathology, College of Food, Agricultural and Environmental Sciences, Ohio State University, Columbus 43210, USA. ${ }^{5}$ Manipal University, Manipal 576104, India. ${ }^{6}$ Genomics Discovery Program, School of Conservation, Life Science and Health Sciences, TransDisciplinary University, Foundation of Revitalization of Local Health Traditions, Bengaluru 560064, India.

Received: 3 November 2015 Accepted: 24 February 2016 Published online: 16 March 2016

\section{References}

1. Goff SA, Ricke D, Lan T-H, Presting G, Wang R, Dunn M, Glazebrook J, Sessions A, Oeller P, Varma H, et al. A Draft Sequence of the Rice Genome (Oryza sativa L. ssp. japonica. Science. 2002;296(5565):92-100.

2. Yu J, Hu S, Wang J, Wong GK, Li S, Liu B, Deng Y, Dai L, Zhou Y, Zhang X, et al. A draft sequence of the rice genome (Oryza sativa L. ssp. indica). Science. 2002;296(5565):79-92.

3. Schatz MC, Maron LG, Stein JC, Wences AH, Gurtowski J, Biggers E, Lee $H$, Kramer M, Antoniou E, Ghiban E, et al. Whole genome de novo assemblies of three divergent strains of rice, Oryza sativa, document novel gene space of aus and indica. Genome Biol. 2014;15(11):506.

4. Sakai H, Kanamori H, Arai-Kichise Y, Shibata-Hatta M, Ebana K, Oono Y, Kurita K, Fujisawa H, Katagiri S, Mukai Y. Construction of pseudomolecule sequences of the aus rice cultivar kasalath for comparative genomics of Asian cultivated rice. DNA Res. 2014;21(4):397-405.

5. Gowda M, Shirke MD, Mahesh HB, Chandarana P, Rajamani A, Chattoo BB. Genome analysis of rice-blast fungus Magnaporthe oryzae field isolates from southern India. Genomics Data. 2015;5:284-91.

6. Krishan A. Rapid flow cytofluorometric analysis of mammalian cell cycle by propidium iodide staining. J Cell Biol. 1975;66(1):188-93.

7. Zerbino DR, Birney E. Velvet: algorithms for de novo short read assembly using de Bruijn graphs. Genome Res. 2008;18(5):821-9.

8. Luo R, Liu B, Xie Y, Li Z, Huang W, Yuan J, He G, Chen Y, Pan Q, Liu Y. SOAPdenovo2: an empirically improved memory-efficient short-read de novo assembler. Gigascience. 2012;1(1):18.

9. Zimin AV, Marçais G, Puiu D, Roberts M, Salzberg SL, Yorke JA. The MaSuRCA genome assembler. Bioinformatics. 2013;29(21):2669-77.

10. Gurevich A, Saveliev V, Vyahhi N, Tesler G. QUAST: quality assessment tool for genome assemblies. Bioinformatics. 2013;29(8):1072-5.

11. English AC, Richards S, Han Y, Wang M, Vee V, Qu J, Qin X, Muzny DM, Reid JG, Worley KC. Mind the gap: upgrading genomes with Pacific Biosciences RS long-read sequencing technology. PLoS One. 2012;7(11), e47768.

12. Xue W, Li J-T, Zhu Y-P, Hou G-Y, Kong X-F, Kuang Y-Y, Sun X-W. L_RNA scaffolder: scaffolding genomes with transcripts. BMC Genomics. 2013;14(1):604.

13. Grabherr MG, Haas BJ, Yassour M, Levin JZ, Thompson DA, Amit I, Adiconis X, Fan L, Raychowdhury R, Zeng Q, et al. Trinity: reconstructing a full-length transcriptome without a genome from RNA-Seq data. Nat Biotechnol. 2011;29(7):644-52.

14. Assefa S, Keane TM, Otto TD, Newbold C, Berriman M. ABACAS: algorithmbased automatic contiguation of assembled sequences. Bioinformatics. 2009;25(15):1968-9.

15. Soderlund C, Nelson W, Shoemaker A, Paterson A. SyMAP: A system for discovering and viewing syntenic regions of FPC maps. Genome Res. 2006;16(9):1159-68.

16. Parra G, Bradnam K, Korf I. CEGMA: a pipeline to accurately annotate core genes in eukaryotic genomes. Bioinformatics. 2007;23(9):1061-7.

17. Campbell MS, Law M, Holt C, Stein JC, Moghe GD, Hufnagel DE, Lei J, Achawanantakun R, Jiao D, Lawrence CJ. MAKER-P: A tool kit for the rapid creation, management, and quality control of plant genome annotations. Plant Physiol. 2014;164(2):513-24.

18. Jones P, Binns D, Chang H-Y, Fraser M, Li W, McAnulla C, McWilliam H, Maslen J, Mitchell A, Nuka G. InterProScan 5: genome-scale protein function classification. Bioinformatics. 2014;30(9):1236-40.

19. Nei M, Gojobori T. Simple methods for estimating the numbers of synonymous and nonsynonymous nucleotide substitutions. Mol Biol Evol. 1986;3(5):418-26. 
20. Zhang Z, Li J, Zhao X-Q, Wang J, Wong GK-S, Yu J. KaKs_Calculator: Calculating Ka and Ks Through Model Selection and Model Averaging. Genomics Proteomics Bioinformatics. 2006;4(4):259-63.

21. Thiel T, Michalek W, Varshney R, Graner A. Exploiting EST databases for the development and characterization of gene-derived SSR-markers in barley (Hordeum vulgare L.). Theor Appl Genet. 2003;106(3):411-22.

22. Schuler GD. Sequence mapping by electronic PCR. Genome Res. 1997;7(5):541-50.

23. Li H, Durbin R. Fast and accurate short read alignment with BurrowsWheeler transform. Bioinformatics. 2009;25(14):1754-60.

24. Li H, Handsaker B, Wysoker A, Fennell T, Ruan J, Homer N, Marth G, Abecasis G, Durbin R, Genome Project Data Processing S. The Sequence Alignment/ Map format and SAMtools. Bioinformatics. 2009;25(16):2078-9.

25. Auwera GA, Carneiro MO, Hartl C, Poplin R, del Angel G, Levy-Moonshine A, Jordan T, Shakir K, Roazen D, Thibault J. From FastQ Data to HighConfidence Variant Calls: The Genome Analysis Toolkit Best Practices Pipeline. Curr. Protoc. Bioinformatics. 2013:11.10. 11-11.10. 33.

26. DePristo MA, Banks E, Poplin R, Garimella KV, Maguire JR, Hartl C, Philippakis AA, del Angel G, Rivas MA, Hanna M. A framework for variation discovery and genotyping using next-generation DNA sequencing data. Nat Genet. 2011;43(5):491-8.

27. Cingolani P, Platts A, le Wang L, Coon M, Nguyen T, Wang L, Land SJ, Lu X, Ruden DM. A program for annotating and predicting the effects of single nucleotide polymorphisms, SnpEff: SNPs in the genome of Drosophila melanogaster strain w1118; iso-2; iso-3. Fly. 2012;6(2):80-92.

28. Miller JR, Koren S, Sutton G. Assembly algorithms for next-generation sequencing data. Genomics. 2010;95(6):315-27.

29. Koren S, Phillippy AM. One chromosome, one contig: complete microbial genomes from long-read sequencing and assembly. Curr Opin Microbiol. 2015;23:110-20

30. Arumuganathan K, Earle ED. Nuclear DNA content of some important plant species. Plant Mol Biol Rep. 1991;9(3):208-18.

31. Temnykh S, DeClerck G, Lukashova A, Lipovich L, Cartinhour S, McCouch S Computational and experimental analysis of microsatellites in rice (Oryza sativa L.): frequency, length variation, transposon associations, and genetic marker potential. Genome Res. 2001:11(8):1441-52.

32. Ma J, Bennetzen JL. Rapid recent growth and divergence of rice nuclear genomes. Proc Natl Acad Sci U S A. 2004;101(34):12404-10.

33. Hayashi K, Yoshida H, Ashikawa I. Development of PCR-based allele-specific and InDel marker sets for nine rice blast resistance genes. Theor App Genet. 2006;113(2):251-60.

34. Hittalmani S, Parco A, Mew T, Zeigler R, Huang N. Fine mapping and DNA marker-assisted pyramiding of the three major genes for blast resistance in rice. Theor Appl Genet. 2000;100(7):1121-8.

35. Sharma T, Rai A, Gupta S, Vijayan J, Devanna B, Ray S. Rice blast management through host-plant resistance: retrospect and prospects. Agricultural Research. 2012;1(1):37-52

36. Chen J, Shi Y, Liu W, Chai R, Fu Y, Zhuang J, Wu J. A Pid3 allele from rice cultivar Gumei2 confers resistance to Magnaporthe oryzae. J Genet Genomics. 2011;38(5):209-16.

37. Das A, Soubam D, Singh P, Thakur S, Singh N, Sharma T. A novel blast resistance gene, Pi54rh cloned from wild species of rice, Oryza rhizomatis confers broad spectrum resistance to Magnaporthe oryzae. Funct Integr Genomics. 2012;12(2):215-28.

38. Hua L, Wu J, Chen C, Wu W, He X, Lin F, Wang L, Ashikawa I, Matsumoto T, Wang $L$. The isolation of Pi1, an allele at the Pik locus which confers broad spectrum resistance to rice blast. Theor Appl Genet. 2012;125(5):1047-55.

39. Ma J, Lei C, Xu X, Hao K, Wang J, Cheng Z, Ma X, Ma J, Zhou K, Zhang X. Pi64, encoding a novel CC-NBS-LRR protein, confers resistance to leaf and neck blast in rice. Mol Plant-Microbe Interact. 2015;28:558-568.

40. Bryan GT, Wu K-S, Farrall L, Jia Y, Hershey HP, McAdams SA, Faulk KN, Donaldson GK, Tarchini R, Valent B. A single amino acid difference distinguishes resistant and susceptible alleles of the rice blast resistance gene Pi-ta. The Plant Cell Online. 2000;12(11):2033-45.

41. Flor HH. Current status of the gene-for-gene concept. Annu Rev Phytopathol. 1971;9(1):275-96.

42. Li J-Y, Wang J, Zeigler RS. The 3,000 rice genomes project: new opportunities and challenges for future rice research. GigaScience. 2014;3(1):1-3.
43. Ballini E, Berruyer R, Morel JB, Lebrun MH, Nottéghem JL, Tharreau D. Modern elite rice varieties of the 'Green Revolution'have retained a large introgression from wild rice around the Pi33 rice blast resistance locus. New Phytol. 2007;175(2):340-50.

44. Hulbert SH, Webb CA, Smith SM, Sun Q. Resistance gene complexes: evolution and utilization. Annu Rev Phytopathol. 2001;39(1):285-312.

45. Yang S, Li J, Zhang X, Zhang Q, Huang J, Chen J-Q, Hartl DL, Tian D. Rapidly evolving $\mathrm{R}$ genes in diverse grass species confer resistance to rice blast disease. Proc Natl Acad Sci. 2013;110(46):18572-7.

46. Padmanabhan S, Mathur S, Misra R. Breeding for blast resistance in India: genetics of blast resistance. Indian J Genet Plant Breed. 1974;34:424-9.

47. Ou SH. Rice diseases. Philippines: IRRI; 1985.

48. Wang G-L, Ruan D-L, Song W-Y, Sideris S, Chen L, Pi L-Y, Zhang S, Zhang Z, Fauquet C, Gaut BS. Xa21D encodes a receptor-like molecule with a leucinerich repeat domain that determines race-specific recognition and is subject to adaptive evolution. Plant Cell. 1998;10(5):765-79.

49. Sun Q, Collins NC, Ayliffe M, Smith SM, Drake J, Pryor T, Hulbert SH. Recombination between paralogues at the rp1 rust resistance locus in maize. Genetics. 2001;158(1):423-38.

\section{Submit your next manuscript to BioMed Central and we will help you at every step:}

- We accept pre-submission inquiries

- Our selector tool helps you to find the most relevant journal

- We provide round the clock customer support

- Convenient online submission

- Thorough peer review

- Inclusion in PubMed and all major indexing services

- Maximum visibility for your research

Submit your manuscript at www.biomedcentral.com/submit
C) BioMed Central 